\title{
Guest Editorial: Trends in Reservoir Computing
}

\author{
Simone Scardapane ${ }^{1} \cdot$ Claudio Gallicchio $^{2} \cdot$ Alessio Micheli $^{2} \cdot$ Miguel C. Soriano $^{3}$
}

(C) The Author(s), under exclusive licence to Springer Science+Business Media, LLC, part of Springer Nature 2021

Reservoir Computing ( $\mathrm{RC})$ is a leading-edge paradigm for the design and training of recurrent neural network models. The approach has become popular among practitioners due to its simplicity of implementation, effectiveness in applications, and efficiency [1]. Theoretically, RC allows to deepen the study of characterizations and initialization of dynamical neural models, improving our understanding of their dynamical behavior. In recent years, studies on neuromorphic implementations of RC have also opened the way to breakthrough advancements enabling ultra-fast learning in the temporal domain [2].

This special issue contains selected papers that provide an overview of the main streams of current research in the $\mathrm{RC}$ field. The papers of this special issue cover topics of neuromorphic computing and non-conventional hardware implementations of RC, optimization of reservoir configurations, theoretical analysis of reservoirs, and emerging developments of RC, including conceptors and deep reservoirs, as well as novel application fields for the RC paradigm. The special issue was preceded by the first international workshop on Reservoir Computing, held at ICANN (September 2019 in Munich, Germany) [3]. The articles have undergone rigorous peer-review according to the journals high standards.

Miguel C. Soriano

miguel@ifisc.uib-csic.es

Simone Scardapane

simone.scardapane@uniroma1.it

Claudio Gallicchio

gallicch@di.unipi.it

Alessio Micheli

micheli@di.unipi.it

1 Department of Information Engineering, Electronics and Telecommunications, Sapienza University of Rome, Rome, Italy

2 Department of Computer Science, University of Pisa, Pisa, Italy

3 Institute for Cross-Disciplinary Physics and Complex Systems, IFISC (CSIC-UIB), Palma de Mallorca, Spain
The Special issue contains eight contributions, which can be grouped in the following categories:

\section{Methodology}

- Efficient Implementations of Echo State Network CrossValidation by M. Lukoševičius et al.

- Latent space exploration and functionalization of a gated working memory model using conceptors by A. Strock et al.

- Echo State Networks and Long Short-Term Memory for Continuous Gesture Recognition: a Comparative Study by D. Jirak et al.

- A Reservoir Computing Approach to Word Sense Disambiguation by K. Simov et al.

\section{Physical Implementations}

- Limitations of the recall capabilities in delay based reservoir computing systems by F. Köster et al.

- Information processing capacity of spin-based quantum reservoir computing systems by $\mathrm{R}$. Martínez-Peña et al.

- Bayesian optimisation of large-scale photonic reservoir computers by P. Antonik et al.

- Hardware-Optimized Reservoir Computing System for Edge Intelligence Applications by A. Morán et al.

These contributions encompass a wide range of research topics, thereby appealing to both the experts in the field and those who want a snapshot of the current breadth of reservoir computing research.

We would like to thank the editor in chief of the journal, Amir Hussain, for the strong support given when organizing and publishing this special issue; all the authors who participated in the issue; and the anonymous reviewers who helped us evaluate and improve the quality of the submissions.

\section{References}

1. Lukoševičius M, Herbert J. Reservoir computing approaches to recurrent neural network training. Comput Sci Rev. 2009;3:127-49. 
2. Tanaka G, Yamane T, Héroux JB, Nakane R, Kanazawa N, Takeda S, Numata H, Nakano D, Hirose A. Recent advances in physical reservoir computing: A review. Neural Netw. 2019;115:100-23.
3. The 1st International Workshop on Reservoir Computing. 2019. https://sites.google.com/view/reservoir-computingworkshop. Accessed 15 Jan 2021. 